\title{
Overview: \\ Deeply bound nuclear states of pions and kaons
}

\author{
Toshimitsu Yamazaki \\ RIKEN, 2-1 Hirosawa, Wako-shi, Saitama, 351-0198 Japan *
}

\begin{abstract}
An overview of the quest for deeply bound states of the two NambuGoldstone bosons (pions and kaons) is given. The deeply bound pionic states, the 1s states in heavy nuclei, serve as a unique probe for chiral symmetry restoration through the isovector s-wave $\pi$-nucleus interaction, as demonstrated in a recent systematic study in Sn isotopes. Narrow nuclear bound states of $\bar{K}$ have been predicted to exist with shrunk non-existent nuclear cores, and some experimental evidences have recently been obtained. They are expected to provide microscopic $\bar{K}$-mediated dense nuclear systems, which are pertinent to kaon condensation without the aid of gravity.
\end{abstract}

\section{From exotic atoms to exotic nuclei}

Studies of exotic mesonic atoms have provided important information on the mesonnucleus potentials. In recent years, more and more attentions have been paid to deeper bound states, ultimately, nuclear ground states involving exotic particles, since they ought to provide more direct information on the interactions. We proceed from traditional "exotic atoms" to "exotic nuclei". In this overview we consider nuclear bound states of two Nambu-Goldstone bosons, $\pi^{-}$and $K^{-}$. In the past, their existence was not expected due to a naive misbelief that they should have too large nuclear absorption widths to be discrete states. Special mechanisms have, however, been pointed out for these mesons to form quasi-stable bound states.

\section{Nuclear systems of the Nambu-Goldstone bosons}

In the case of $\pi^{-}$Coulomb assisted bound states were shown to be quasi-stable; the shortrange repulsive potential together with a long-range Coulomb attraction can accommodate

\footnotetext{
${ }^{*}$ E-mail address: yamazaki@nucl.phys.s.u-tokyo.ac.jp
} 
halo like bound states with partial overlapping with the nucleus $[1,2]$. The presence of narrow bound states was indeed demonstrated by the first observation of deeply bound $\pi^{-}$states in ${ }^{207} \mathrm{~Pb}$ in 1996 [3], and subsequent experimental studies followed [4, 5].

In the case of $K^{-}$bound states, narrow nuclear bound states were predicted by Akaishi et al. $[6,7,8,9,10,11]$ by a different mechanism; namely, the strongly attractive force of $\bar{K} N$ which they deduced from empirical data produces deeply bound states below the threshold of $\Sigma \pi$ emission, which is the main decay mode of $\bar{K} N$ in nuclei, and thus their decay widths are reduced to around $20 \mathrm{MeV}$. They calculated few-body bound states including a single and double $\bar{K}$ 's, starting from the ansatz that the $\Lambda(1405)$ resonance is a $I=0$ bound state of $\bar{K} N$. Very recently, some experimental evidences have been observed $[12,13]$ (see Iwasaki of this volume [14]). Thus, it is timely to discuss this new aspect of physics.

The bare $\pi N$ and $\bar{K} N$ interactions, which are obtained from empirical data (low energy scattering lengths, mesonic hydrogen and deuterium data), can be understood from the Tomozawa-Weinberg relations $[15,16]$ between the $T$ matrices and the meson decay constants $\left(f_{\pi} \sim f_{K} \sim 90 \mathrm{MeV}\right)$ :

$$
\begin{aligned}
T_{\pi^{-} p}=-T_{\pi^{-} n} & =\frac{m_{\pi}}{f_{\pi}^{2}}, \\
T_{K^{-} p}=-T_{K^{+} p} & =\frac{m_{K}}{f_{K}^{2}}, T_{K^{-} n}=-T_{K^{+} n}=\frac{m_{K}}{2 f_{K}^{2}},
\end{aligned}
$$

The pion decay constant is connected to the quark condensate in vacuum by the GOR relation [17]. These $T$ matrices are expected to change in nuclear medium, where the chiral symmetry is partially restored and the quark condensate is decreased.

The repulsive nature of the isovector part of the s-wave $\pi N$ interaction, as given above, is in good agreement with observed free $\pi p$ and $\pi n$ interactions. The study of $\pi$-nucleus interaction will inform how the $\pi N$ interaction is modified in the nuclear medium [18].

The Tomozawa-Weinberg relation also indicates that the $K^{-} p$ interaction is very strong. This problem was more elaborated by a chiral SU(3) theory [19] (see also Weise in this volume [20]). The strong attraction is also understood by a meson exchange theory [21], which shows an additive role of $\sigma-\rho-\omega$ exchange in the case of $\bar{K} N$ interaction because of the nature of particle-antiparticle interactions, in contrast to the baryon-baryon interactions.

\section{Deeply bound pionic states as a probe for chiral symmetry restoration}

The 1s ground states in heavy nuclei are the best in deducing the isovector part (so called $b_{1}$ parameter) of the s-wave part of the pion-nucleus interaction, which is related to partial restoration of the quark condensate in nuclear medium [18]. A recent experiment on pionic bound states in Sn isotopes has revealed that the observed $b_{1}$ parameter is enhanced over the free value:

$$
R=\frac{b_{1}^{\text {free }}}{b_{1}^{\text {obs }}}=0.78 \pm 0.05 \approx \frac{b_{1}^{\text {free }}}{b_{1}^{*}\left(\rho_{e}\right)} \approx \frac{f_{\pi}^{*}\left(\rho_{e}\right)^{2}}{f_{\pi}^{2}} \approx 1-\alpha \rho_{e}
$$


where $\rho_{e} \approx 0.60 \times \rho_{0}$ is the effective nuclear density at the bound-pion location and $\alpha$ is determined to be $0.36 / \rho_{0}$. Thus, the observed enhancement of $\left|b_{1}\right|$ over the free $\pi N$ value implies that the chiral order parameter, $f_{\pi}^{*}(\rho)^{2}$, would be reduced by a factor of $\approx 0.64$ at the normal nuclear density $\rho=\rho_{0}$.

Whether or not the $\bar{K} N$ interaction is enhanced in nuclear medium is an unsolved important issue. We can address this question by observing the predicted $\bar{K}$-bound states.

\section{Deep and narrow kaonic bound states predicted: non-existing shrunk nuclear cores}

Since the $I=0 \bar{K} N$ interaction is strongly attractive without hard core in short range, a $\bar{K}$ is able to form very unusual condensed nuclear systems, which do not exist in nature, as shown in Ref. [6, 7, 8, 9, 10, 11]. For example, Fig. 1, a result of Antisymmetrized Molecular Dynamics calculation [8,9], shows dramatically how the ${ }^{8}$ Be nucleus which is composed of two alpha clusters is shrunk by adding a $K^{-}$meson. It is interesting to see that the condensed system appears to be composed of nearly two dense "mini alpha" clusters. Such dense $\bar{K}$-bound states deserve a name of " $\bar{K}$ cluster".

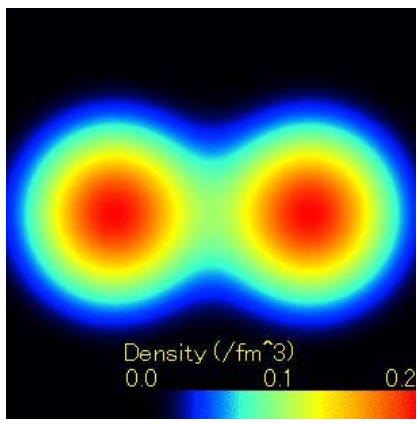

(a) ${ }^{8} \mathrm{Be}$

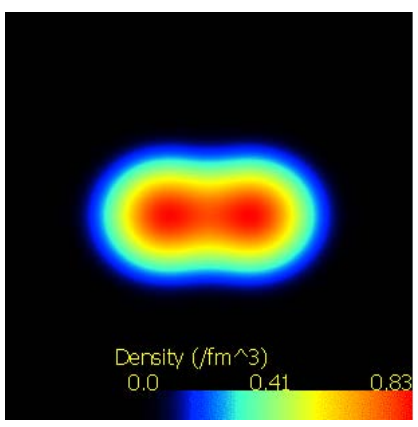

(b) ${ }^{8} \mathrm{BeK}^{-}$

Figure 1: Comparison of the calculated density contours of (a) ordinary ${ }^{8} \mathrm{Be}$ and (b) ${ }^{8} \mathrm{BeK}^{-}$, shown in the size of 7 by $7 \mathrm{fm}[8]$.

The results of calculation for various exotic nuclear cores, which do not exist without $\bar{K}$, are listed in Table 1 . These results are based on the following procedures. i) The bare $\bar{K} N$ interactions, which were derived by coupled-channel calculations from the low-energy $\bar{K} N$ data [6], are applied to few-body systems without any model assumptions. ii) The $N N$ hard core was taken into account by using a realistic $N N$ scattering data at high energy. The average nucleon densities amount to $\sim 3 \times \rho_{0}$. This peculiar situation does not allow at all a description of $\bar{K}$-bound systems in terms of a common global potential with known nuclear size parameters. See also Akaishi [22] and Doté [23] of this volume. It is our strategy to obtain new information on the $\bar{K} N$ interaction in medium by comparing the calculated $\bar{K}$ binding energies with forthcoming observation. 
Table 1: Summary of predicted $\bar{K}$ clusters. $M$ : total mass $[\mathrm{MeV}] .-E_{K}$ : total binding energy $[\mathrm{MeV}] . \Gamma_{K}$ : decay width $[\mathrm{MeV}] . \rho(0)$ : nucleon density at the center of the system $\left[\mathrm{fm}^{-3}\right] . R_{\mathrm{rms}}$ : root-mean-square radius of the nucleon system $[\mathrm{fm}]$. These data are from the earlier calculations $[6,7,8,9,10]$. In view of recent experimental observations the binding energy values will be subject to increase due to various causes [11].

\begin{tabular}{l|ccccc}
\hline$K$ cluster & $\begin{array}{c}M c^{2} \\
{[\mathrm{MeV}]}\end{array}$ & $\begin{array}{c}-E_{K} \\
{[\mathrm{MeV}]}\end{array}$ & $\begin{array}{c}\Gamma_{K} \\
{[\mathrm{MeV}]}\end{array}$ & $\begin{array}{c}\rho(0) \\
{\left[\mathrm{fm}^{-3}\right]}\end{array}$ & $\begin{array}{c}R_{\text {rms }} \\
{[\mathrm{fm}]}\end{array}$ \\
\hline \hline$p K^{-}$ & 1407 & 27 & 40 & 0.59 & 0.45 \\
$p p K^{-}$ & 2322 & 48 & 61 & 0.52 & 0.99 \\
$p^{-} p K^{-}$ & 3211 & 97 & 13 & 1.56 & 0.81 \\
$p^{-} n K^{-}$ & 3192 & 118 & 21 & 1.50 & 0.72 \\
ppppK $^{-}$ & 4171 & 75 & 162 & 1.68 & 0.95 \\
pppn $K^{-}$ & 4135 & 113 & 26 & 1.29 & 0.97 \\
$p p n n K^{-}$ & 4135 & 114 & 34 & & 1.12 \\
\hline$p p K^{-} K^{-}$ & 2747 & 117 & 35 & & \\
ppn $K^{-} K^{-}$ & 3582 & 221 & 37 & 2.97 & 0.69 \\
pppn $K^{-} K^{-}$ & 4511 & 230 & 61 & 2.33 & 0.73 \\
\hline
\end{tabular}

\section{Deeply bound kaonic states observed: even more deeply}

In 2004 a KEK experiment on the ${ }^{4} \mathrm{He}\left(\right.$ stopped- $\left.K^{-}, p\right)$ process revealed a distinct state, which was name "strange tribaryon $\mathrm{S}^{0}(3150)$ " [13] (see Iwasaki of this volume [14]). In the hadronic quantum number this corresponds to a bound state of $T=1 \mathrm{pnnK} K^{-}$, which turned out to be one of the $\left(T, T_{3}\right)=(1,-1)$ isobaric analog state of the already predicted $\left(T, T_{3}\right)=(1,+1) p p p K^{-}[7,9]$. Surprisingly, the observed binding energy $(193 \mathrm{MeV})$ was substantially larger than the predicted value $(97 \mathrm{MeV})$. This $T=1$ family was predicted to be formed with a non-existing nuclear core $[N N N]_{T=3 / 2}$, in which one of the nucleon is flipped to an excited orbital $0 p_{3 / 2}$ (see the density distributions in the top of Fig. 2). This is not to be confused with another $T=1$ member with a "triton-like" core with $T=1 / 2$, which was predicted to be shallow lying and broad. On the other hand, the originally predicted $T=0 p p n K^{-}$state with a binding energy of $111 \mathrm{MeV}$ was searched for in the ${ }^{4} \mathrm{He}$ (stopped- $\left.K^{-}, n\right)$ process, and some evidence was reported [12]. Its binding energy was found to be $169 \mathrm{MeV}$, slightly smaller than the $T=1$ member, but again, much deeper than the prediction.

These KEK experiments demonstrated that deep and narrow bound states indeed exist. The surprisingly large binding energies of these states were consistently accounted for [11] by taking account the numbers of the $I=0 \bar{K} N$ pairs in these states, relativistic effects and enhanced $\bar{K} N$ interactions in dense medium. Figure 2 illustrates the situation.

It should be noted that the core energy $\Delta E_{\text {core }}$ is a very large compression energy because of the shrinkage, and thus, the deduced net $\bar{K}$-nucleus potential is deeper by this 


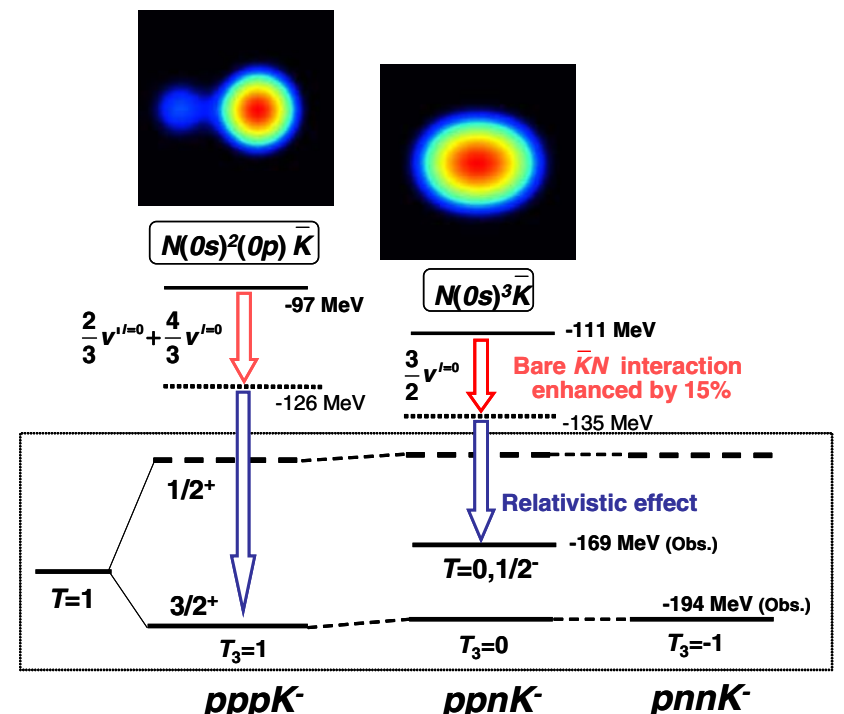

Figure 2: Spin-isospin structure of the strange tribaryon system $[(N N N) \bar{K}]_{\left(T, T_{3}\right)}^{Q}$. The previously calculated nucleon-density contours and energy levels with $E_{K}$ values are shown on top. The observed $S^{0}(3115)$ and $S^{1}(3140)$ are identified as the $\left(T, T_{3}\right)=(1,-1)$ and $T=0$ states, respectively. The experimental energies are accounted for by the relativistic effect and by invoking medium-modified bare $\bar{K} N$ interactions with an enhancement of 1.15. A large spin-orbit splitting is expected for each $T=1$ triplet member. From [11].

amount, as shown in Fig. 3. In this situation the relativistic correction becomes large, pushing the level downward. However, the corrected energy is still insufficient to account for the observed value. We need an enhanced bare $\bar{K} N$ interaction strength by about 15 $\%$ [11]. The unusually large spin-orbit splitting in this dense system, $E_{p 1 / 2}-E_{p 3 / 2}=60$ $\mathrm{MeV}$, contributes to lowering the $T=1$ energy.

Why can such an exotic "core-excited" state be populated in the stopped- $K^{-}$reactions? The key to understand this is the well known role of the $\Lambda(1405)$ as a doorway in the $K^{-}$ nuclear absorption $[7,11]$.

An independent experiment with FINUDA [24] has revealed an indication of a bound $p p K^{-}$state at around $80-120 \mathrm{MeV}$. This is also larger than the original prediction of 60 $\mathrm{MeV}$ [7]. Applying the enhancement factor of 1.15 we predict a new value of $80 \mathrm{MeV}$.

There is a preliminary data of ${ }^{16} \mathrm{O}\left(K^{-}, n\right)$ [25], which also shows a deep bound state. 


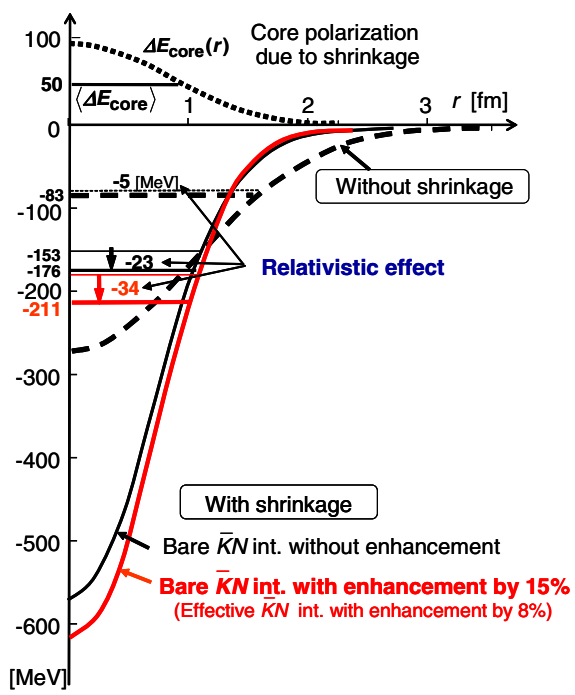

Figure 3: Calculated $\bar{K}$-core potentials, $U_{\bar{K} \text {-core }}(r)$, for the $T=0 p p n K^{-}$state in three cases: without shrinkage, with shrinkage with the original bare $\bar{K} N$ interaction and with an enhanced bare $\bar{K} N$ interaction by $15 \%$. The relativistic effects on the $\bar{K}$-core binding energies $\left(E_{\bar{K} \text {-core }}\right)$ are indicated by arrows. Also shown is the nuclear core energy, $\Delta E_{\text {core }}(r)$, with an average value $\left(<\Delta E_{\text {core }}>\right)$. From $[11]$.

\section{Possible experimental signatures for core shrinkage}

Deeply bound $\bar{K}$ nuclear states in few-body systems are theoretically expected only when the nuclear cores are shrunk. However, we need direct experimental evidences for the shrinkage. In Ref. [11] two possible signatures are mentioned. One is the Coulomb energy displacements in the $T=1$ triplet states. Another is the spin-orbit splitting of the $0 p_{3 / 2}-$ $0 p_{1 / 2}$ doublet, which is expected to be $60 \mathrm{MeV}$ in the dense system, whereas it is only a few $\mathrm{MeV}$ in a normal system. As a more direct signature one can measure the momentum correlation of three decay particles from a $\bar{K}$-bound state, such as $T=0 p p n K^{-}$and $T=1 p p p K^{-}[26]$. This information allows also the assignment of the angular momentum of the parent state. For this purpose we have to explore more experimental methods for the production of $\bar{K}$-bound states.

\section{Direct reactions and compound reactions}

It is vitally important to produce more exotic $\bar{K}$-bound states. Since the $\bar{K}$ nuclear states are regarded as Feshbach resonances in the $S=-1$ sector, various strangeness-exchange direct reactions, such as $\left(K^{-}, \pi\right),\left(\pi^{+,-}, K^{+, 0}\right),\left(\gamma, K^{+, 0}\right)$ and $\left(K^{-}, N\right)$ reactions, can pro- 
Table 2: Various reactions to produce exotic $\bar{K}$-bound systems

\begin{tabular}{l|cc|ccc|cc}
\hline & $\left(K^{-}, p\right)$ & $\left(K^{-}, n\right)$ & $\left(\pi^{-}, K^{0}\right)$ & $\begin{array}{c}\left(K^{-}, \pi^{-}\right) \\
\left(\pi^{+}, K^{+}\right)\end{array}$ & $\left(\pi^{+}, K^{0}\right)$ & $\left(p, K^{+}\right)$ & $\left(p, K^{0}\right)$ \\
\hline$\Delta Q$ & -2 & -1 & -1 & 0 & +1 & 0 & +1 \\
\hline \hline Target & & & \multicolumn{5}{c}{} \\
\hline$p$ & - & - & $\Lambda, \Lambda^{*}$ & $\Sigma^{+}, \Sigma^{+*}$ & - & $p p K^{-}$ & - \\
{$[n]$} & - & - & $\Sigma^{-}, \Sigma^{-*}$ & $p n K^{-}$ & $p p K^{-}$ & & \\
$d$ & - & $\Lambda, \Lambda^{*}$ & $p n K^{-}$ & $p p K^{-}$ & - & $p p n K^{-}$ & $p p p K^{-}$ \\
${ }^{3} \mathrm{He}$ & $p n K^{-}$ & $p p K^{-}$ & $p p n K^{-}$ & $p p p K^{-}$ & - & $p p p n K^{-}$ & $p p p p K^{-}$ \\
${ }^{4} \mathrm{He}$ & $p n n K^{-}$ & $p p n K^{-}$ & $p p n n K^{-}$ & $p p p n K^{-}$ & $p p p p K^{-}$ & $p p p n n K^{-}$ & $p p p n K^{-}$ \\
\hline
\end{tabular}

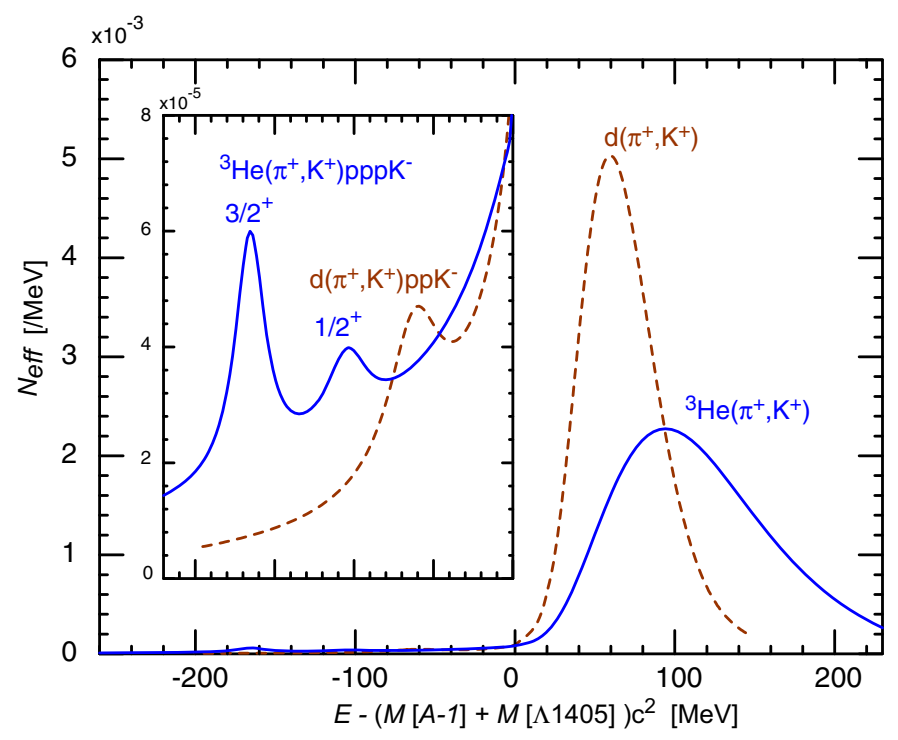

Figure 4: Spectra $\left(N_{\text {eff }}\right)$ of $\left(\pi^{+}, K^{+}\right)$reactions at $p_{\pi}=1.5 \mathrm{GeV} / c$ on $d$ and ${ }^{3} \mathrm{He}$ as functions of $E_{K}-\left[M(A-1)+M\left(\Lambda_{1405}\right)\right] c^{2}$, calculated based on the $\Lambda^{*}$ doorway model. Not only the $J^{\pi}=3 / 2^{+}$state, but also its spin-orbit partner $\left(1 / 2^{+}\right)$with a splitting of $60 \mathrm{MeV}$ are incorporated.

duce many different species $[6,7,27]$, as shown in Table 2. Not only the direct process but also reactions through continumm (compound states) will contribute to their productions. The FINUDA case [24] is one of those. When missing mass spectroscopy is not possible, invariant mass spectroscopy may be useful. Example of expected spectral shapes are shown in Fig. 4, where the binding energies are taken from the recent observation. 
Predicted spin-orbit partners in the case of $T=1 \mathrm{ppp} K^{-}$are indicated.

More recently, nuclear reactions, such as $p+d$ and $p+$ nucleus, have been considered to use. See Suzuki of this volume [28].

\section{Double $\bar{K}$ systems}

Double $\bar{K}$ nuclei, such as $p p K^{-} K^{-}, p p n K^{-} K^{-}$and $p p p K^{-} K^{-}$, are predicted to exist even with larger binding energies, almost twice as much as in the single $\bar{K}$ systems [7, 10]. Now that the observed single- $\bar{K}$ systems show large binding energies than in the original prediction we can safely presume that the binding energies of double- $\bar{K}$ systems are around $400 \mathrm{MeV}$. How can we produce those? Besides the $\left(K^{-}, K^{+}\right)$type reactions (and through their continuum compound reactions) we propose to search for those in heavy-ion reactions at high energy [10]. First, a cascade evolution of $\bar{K}$ clusters (capture reactions), in which the $\bar{K}$ falls into a deeper and deeper state by capturing surrounding nucleons like a self-trapping center, may take place. Secondly, Fig. 5 shows a dense $\bar{K}$ cluster formed in a hot plasma remains in a cooling stage, like a microscopic solid residue in liquid/gas. To identify $\bar{K}$ clusters we employ invariant-mass spectroscopy method, which is applicable when all the decay channels can be measured and the decay occurs after the freeze-out phase.

\section{Quark Gluon Plasma}
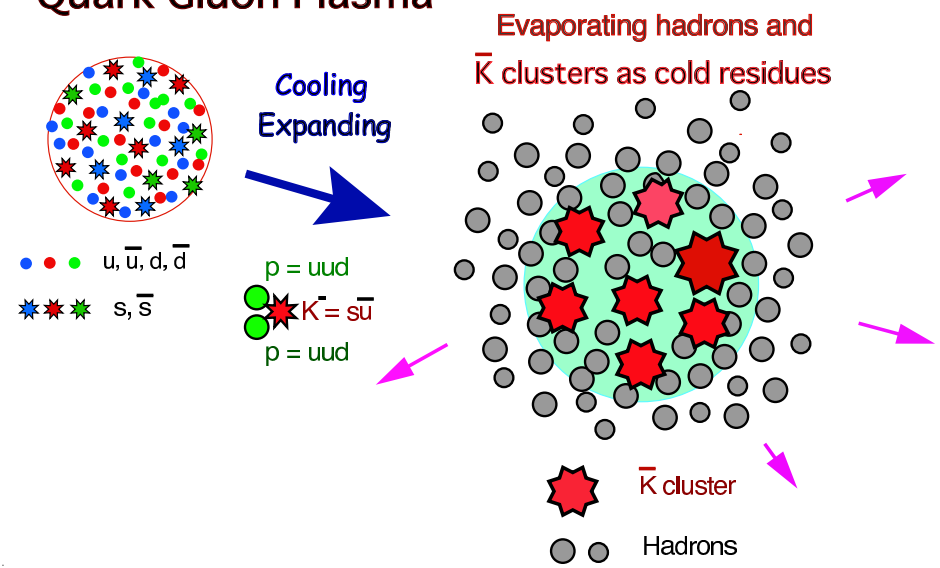

Figure 5: Quark gluon plasma and its transition to evaporating hadron gases with heavy and dense residues of $\bar{K}$ clusters.

A preliminary result is available from the FOPI group at GSI (see Herrmann of this volume [29]). They analyzed the data of $\mathrm{Ni} \times \mathrm{Ni}$ reactions at $2 \mathrm{GeV} / \mathrm{u}$ and reconstructed an invariant-mass spectrum of $\Lambda+d$. The spectrum seems to show a small but significant 
peak over a huge combinatorial background, which must correspond to a $T=0$ ppn $K^{-}$ cluster. It is intriguing that the peak energy of about $3.16 \mathrm{GeV}$ seems to agree with the reported value from KEK [12].

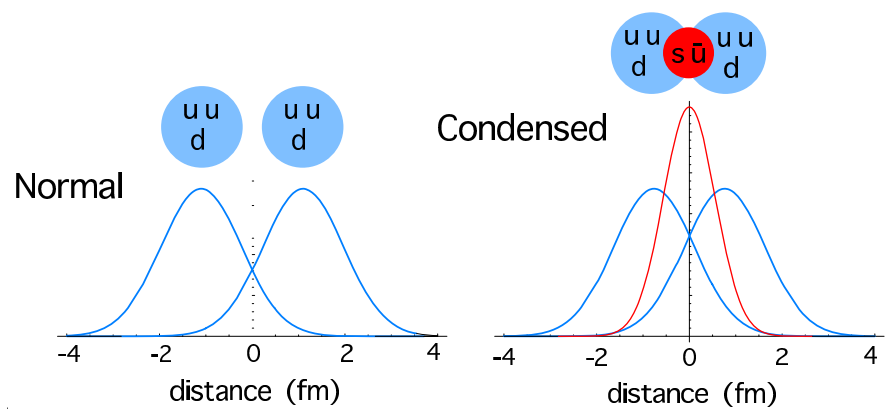

Figure 6: (Left) Two neighbouring nucleons with their finite density distributions $\left(r_{\text {rms }} \sim\right.$ $0.86 \mathrm{fm}$ ) and their average $N-N$ distance of $d_{N N} \sim 2.2 \mathrm{fm}$ in nornal nuclei with density $\rho_{0} \sim 0.17 \mathrm{fm}^{-3}$. (Right) Two nucleons with a reduced $N-N$ distance of $d_{N N} \sim 1.6 \mathrm{fm}$, realized by an intruding $\bar{K}$ to produce a dense nuclear system with $\left\langle\rho>\sim 3 \rho_{0}\right.$.

\section{Why does $\bar{K}$ produce shrunk nuclear systems?}

Why are such high-density nuclear states possible against the nuclear physics "law" of constant nuclear density? The constant density of nuclei is kept valid by the hard core part of the $N-N$ interaction, which may result from the Pauli blocking in the $u-d$ quark sector. This is shown in Fig. 6 (Left). Usual hadrons are subject to short-range repulsion because of the $(u, d)$ Pauli blocking. In normal nuclei the average inter-nucleon distance is $d_{N N} \sim 2.2 \mathrm{fm}$. The nucleon rms radius, $r_{\mathrm{rms}} \sim 0.86 \mathrm{fm}$, corresponds to a nucleon volume of $v_{N} \sim 2.66 \mathrm{fm}^{3}$ and to a nucleon density of $\rho_{N} \sim 0.38 \mathrm{fm}^{-3}$. This means that nucleons occupy the nuclear space with a compaction factor of $f_{c}=\rho_{N} / \rho_{0} \sim 2.3$. This situation is hardly changed.

The exceptional case of the $\bar{K}$ meson, composed of $s \bar{u}\left(\bar{K}^{0}\right)$ or $s \bar{d}\left(K^{-}\right)$, which includes no $(u, d)$ quark. Thus, the $\bar{K} N$ interaction is dominated by the particle-antiparticle attraction without hard core. (A similar exceptional situation exists for $D$ mesons). From this consideration we understand why normal nuclei are difficult to compress and why only the $\bar{K}$ meson mediates dense nuclear systems. The $\bar{K}$ meson is an intruder to relax the $N N$ hard core, thus increasing the average nucleon density to $<\rho>\sim 3 \rho_{0}$, which exceeds the above nucleon compaction factor (Fig. 6 (Right)) at which the QCD vacuum is expected to vanish and chiral symmetry is restored. It is vitally important to investigate to what extent the involved hadrons keep their identities under such an extremely dense system. 

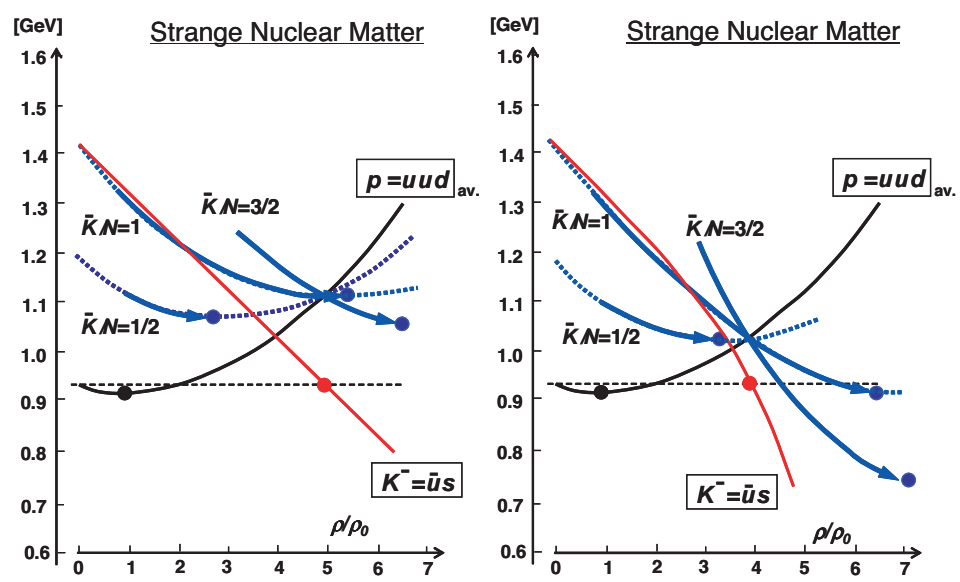

Figure 7: Speculated diagrams for the density dependences of the bound-state energies of various baryon composite systems $\left(p K^{-}\right)^{m} \mathrm{n}^{n}$. The $\bar{K} N$ energy is represented by the red line/curve, and the nuclear compression by the black curve. The total energies for representative fractions of $K^{-} / N(=1 / 2,1$ and 3/2) are depicted by respective blue curves, showing minima at high density and low energy. (Left) In the case of density-independent $\bar{K} N$ without relativistic correction. (Right) In the case of density-dependent enhanced $\bar{K} N$ with relativistic correction.

In this respect, $\bar{K}$ clusters are viewed as particle-antiparticle systems, which, however, are not as violent as in $\bar{p}$ nuclear systems.

\section{Dense nuclear systems and kaon condensation}

The above consideration naturally leads us to a regime of kaon condensation [30, 31]. Namely, $\bar{K}$ mesons, as intruders with $\bar{u}$ and $\bar{d}$ quarks, behave as strong glue to combine surrounding nucleons to a dense system. The whole energy drops down, depending on the composition of $p, n$ and $\bar{K}$. Intuitively, one can conceive energy diagrams, such as Fig. 7 .

So, the microscopic $\bar{K}$-bound nuclear clusters, which we are studying now, are building blocks of large Kaon Matter. Not only does their existence itself have fundamental importance from the viewpoint of dense and bound quark-gluon systems, but also is pertinent to the problem of strange matter and star.

The author would like to thank Drs. Y. Akaishi, A. Doté, P. Kienle, M. Iwasaki and N. Herrmann for daily stimulating discussion and collaboration. 


\section{References}

[1] E. Friedman and G. Soff, J. Phys. G 11 (1985) L37.

[2] H. Toki and T. Yamazaki, Phys. Lett. B 213, 129 (1988); H. Toki, S. Hirenzaki, T. Yamazaki, and R.S. Hayano, Nucl. Phys. A 501, 653 (1989).

[3] T. Yamazaki it et al., Z. Phys. A 355, 219 (1996).

[4] H. Geissel et al., Phys. Rev. Lett. 88, 122301 (2002).

[5] K. Suzuki et al., Phys. Rev. Lett. 92 (2004) 072302.

[6] Y. Akaishi and T. Yamazaki, Phys. Rev. C 65 (2002) 044005.

[7] T. Yamazaki and Y. Akaishi, Phys. Lett. B 535 (2002) 70.

[8] A. Doté, H. Horiuchi, Y. Akaishi and T. Yamazaki, Phys. Lett. B 590 (2004) 51.

[9] A. Doté, H. Horiuchi, Y. Akaishi and T. Yamazaki, Phys. Rev. C 70 (2004) 044313.

[10] T. Yamazaki, A. Doté and Y. Akaishi, Phys. Lett. B 587 (2004) 167.

[11] Y. Akaishi, A. Doté and T. Yamazaki, Phys. Lett. B 613 (2005) 140.

[12] M. Iwasaki et al., arXiv:nucl-ex/0310018.

[13] T. Suzuki et al., Phys. Lett. B 597 (2004) 263.

[14] M. Iwasaki, this volume.

[15] Y. Tomozawa, Nuovo Cimento A 46 (1966) 707.

[16] S. Weinberg, Phys. Rev. Lett. 17 (1966) 616.

[17] M. Gell-Mann, R.J. Oakes and B. Renner, Phys. Rev. 175, 2195 (1968).

[18] P. Kienle and T. Yamazaki, Prog. Part. Nucl. Phys. 52 (2004) 85.

[19] T. Waas, N. Kaiser, and W. Weise, Phys. Lett. B 365 (1996) 12; Phys. Lett. B 379 (1996) 34; N. Kaiser, P. B. Siegel, and W. Weise, Nucl. Phys. A594 (1995) 325; W. Weise, Nucl. Phys. A610 (1996) 35.

[20] W. Weise, this volume.

[21] A. Müller-Groeling, K. Holinde and J. Speth, Nucl. Phys. A 513 (1990) 557.

[22] Y. Akaishi, this volume.

[23] A. Doté, this volume.

[24] FINUDA data. T. Nagae, this volume; M. Agnello et al., Phys. Rev. Lett. 94 (2005) 212303.

[25] T. Kishimoto et al., Nucl. Phys. A 754 (2005) 383.

[26] P. Kienle, Y. Akaishi and T. Yamazaki, to be published.

[27] T. Kishimoto, Phys. Rev. Lett. 83 (1999) 4701.

[28] K. Suzuki, this volume.

[29] N. Herrmann, this volume.

[30] D.B. Kaplan and A.E. Nelson, Phys. Lett. B175 (1986) 57.

[31] G.E. Brown, C.H. Lee, M. Rho and V. Thorsson, Nucl. Phys. A567 (1994) 937; G.E. Brown, ibid. A574 (1994) 217c; G.E. Brown and M. Rho, Phys. Rep. 269 (1996) 333. 
